\title{
The Algorithm with Multiple Genotypes on Optimal Warfarin Doses in Korean Patients
}

\author{
Jia-Xin Li, ${ }^{1,2}$ Moo Hyun Kim, ${ }^{1 *}$ Jin-Yeong Han, ${ }^{3, *}$ Kai Song, ${ }^{1}$ EnZe Jin, ${ }^{2}$ LongZhe Guo, ${ }^{1,4}$ Soo Jin Kim, ${ }^{1}$ Kang Min Lee, ${ }^{1}$ Victor Serebruany ${ }^{5}$ \\ 'Department of Cardiology, Dong-A University Medical Center, Busan, Korea \\ 2Department of Cardiology, Harbin Medical University Fourth Hospital, China \\ ${ }^{3}$ Department of Laboratory Medicine, Dong-A University Hospital, Busan, Korea \\ ${ }^{4}$ Department of Cardiology, The First Hospital of Qiqihaer City, Qiqihaer, China \\ ${ }^{5}$ Johns Hopkins University, Baltimore, Maryland, USA
}

Received: 21 April 2020

Accepted: 9 May 2020

*Corresponding author:

Moo Hyun Kim, MD, FACC

Department of Cardiology, College of

Medicine, Dong-A University, 26

Daeshingongwon-ro, Seo-gu, Busan

49201, Korea

Tel: +82-51-240-2976

Fax: +82-51-255-2177

E-mail: kimmh@dau.ac.kr

*Co-correspondence to:

Jin-Yeong Han, MD

Department of Laboratory Medicine,

College of Medicine, Dong-A University,

26 Daeshingongwon-ro, Seo-gu, Busan

49201, Korea

Tel: +82-51-240-2976

Fax: +82-51-255-2177

E-mail: jyhan@dau.ac.kr

Dr. MH Kim received a research grant from Bayer co. Other authors did not have any conflict.

Copyright (๑) Korean Society on Thrombosis and Hemostasis. All rights reserved.
Background and Objectives: Genetic factors that affect warfarin dose are not routinely evaluated in the Korean population. In this study, we investigated the influence of genetic polymorphisms (GPs) on optimal warfarin dose (OWD) and derived an OWD prediction algorithm based on Korean patients with various diseases requiring anticoagulation therapy.

Methods: One hundred eight patients taking warfarin were included. We evaluated clinical characteristics, OWD, international normalized ratio (INR), VKORC1, CYP2C9, and CYP4F2 polymorphisms, as well as medication information. OWD was defined as the maintenance dose that kept a patient's INR within the target range based on at least two consecutive laboratory measurements separated by more one 1 week.

Results: The 108 patients (mean age: $61.5 \pm 12.4 \mathrm{yr}, 48 \%$ male) had a mean OWD of $3.12 \pm 1.30(1-9) \mathrm{mg} /$ day. VKORC1 wild-type patients (AA) had a lower OWD than VKORC1 variant patients (GA). Significantly more OWD patients had the CYP2C9 wild-type genotype than CYP2C9 mutant genotypes. Among the three genotypes of CYP4F2, two carriers had a significantly higher OWD than patients who had the wildtype genotype. We derived an OWD algorithm that included VKORC1, CYP2C9, CYP4F2, body mass index (BMI), age, amiodarone use, and diuretic use.

Conclusion: Our algorithm was capable of explaining $41.8 \%$ of the total variation in warfarin dose in our patient cohort. Multiple GPs affect the OWD in Korean patients.

Keywords: algorithm, optimal warfarin dose, VKORC1, CYP2C9, CYP4F2

\section{Introduction}

Warfarin is widely prescribed to prevent and treat thromboembolic diseases such as atrial fibrillation (AF), deep vein thrombosis (DVT), and pulmonary thromboembolic disease (PTE). ${ }^{1}$ However, multiple challenges making it difficult to determine the optimal dose of warfarin including the long half-life of warfarin, numerous foods that interfere with the actions of warfarin, and drug interactions. ${ }^{2}$ To ensure a suitable level of anticoagulation, prothrombin time (PT) standardized by the international normalized ratio (INR) should be monitored closely.

Cytochrome P-450 2C9 (CYP2C9) and vitamin K epoxide reductase complex subunit 1 (VKORC1) genetic polymorphisms (GPs) affect warfarin pharmacodynamics, ${ }^{3}$ and the product label of warfarin encourages genotype-guided dosing. ${ }^{4}$ Warfarin is a mixture of Rand S-warfarin. S-warfarin is about 3- to 5-fold more effective than $\mathrm{R}$-warfarin at anticoagulation. CYP2C9 is the main enzyme that metabolizes S-warfarin. Active drug can be biotransformed by the CYP2C9 isoenzyme to an inactive metabolite, and the presence of loss-of-function polymorphisms leads to a higher active drug con- centration. Therefore, CYP2C9 polymorphisms affect warfarin dose. ${ }^{3}$ VKORC1 is a warfarin-sensitive and rate-limiting enzyme. Warfarin exerts its anticoagulant effects by inhibiting VKORC1 to affect vitamin K circulation. A common VKORC1 variant $(1639 \mathrm{G}>\mathrm{A})$ has decreased gene expression. Consequently, patients with this VKORC1 variant have different warfarin dose requirements than patients with wild-type VKORC $1 .^{5}$ GPs in CYP2C9 and VKORC1 combined with non-genetic factors were able to explain $45 \%$ of the variance in individual warfarin dose in Chinese patients, ${ }^{6}$ but $55 \%$ of the variance remained unexplained. Cytochrome P-450 4F2 (CYP4F2) is a vitamin $\mathrm{K} 1$ oxidase that affects warfarin dose. Adding this factor could account for more of the variation among patients in optimal warfarin dose, but the effect of CYP4F2 on warfarin dose is controversial as although one study found that CYP4F2 genotype significantly affected warfarin dose, ${ }^{7}$ other studied reported the opposite. ${ }^{8,9}$

Ethnic differences have a large influence on OWD. Asian patients have lower warfarin dose requirements than patients of other ethnicities. ${ }^{10}$ Warfarin dose prediction algorithms that were derived based on patients of other ethnicities may therefore not be suitable for Korean patients. Our aim in this study was to determine the effects of 
various GPs on OWD in Korean patients and derive a warfarin dose prediction algorithm for Korean patients. To improve the overall predictability of our algorithm, we included the CYP4F2 gene in our algorithm.

\section{Methods}

Study design

One hundred eight Korean patients taking warfarin were enrolled in the this study from July 2007 to June 2018. Inclusion criteria were patients who were between 20-80 years old with a body weight above $50 \mathrm{~kg}$ taking warfarin. Exclusion criteria were a history of chronic liver failure, use of other anticoagulant medications, active malignancy, renal disease (creatinine $>2.0 \mathrm{mg} / \mathrm{dL}$ or eGFR $<45 \mathrm{~mL} / \mathrm{min}$ ), or life expectancy $<1$ year.

All patients provided written informed consent prior to participating in the study. We collected clinical data by reviewing patients' charts and electronic medical records, from outpatient clinic visits, as well as by telephone. Data included sex, age, height, body weight, smoking, alcohol, target INR, comorbidities, concurrent medications, left ventricular ejection fraction (LVEF), and OWD. Main indications for treatment were atrial fibrillation (AF), DVT/PTE, and heart valve disease (HVD). Comorbidities were cerebral infarction (CI), congestive heart failure (CHF), hypertension, diabetes mellitus (DM), and hyperlipidemia (HLP). Concurrent medications were $\beta$-blockers, amiodarone, angiotensin receptor blockers (ARBs), angiotensin-converting enzyme inhibitors (ACEi), aspirin, clopidogrel, statins, calcium channel blockers (CCBs), diuretics, and nitrates. The concomitant medications were included when the patients were continuously taking these for at least 7 days during treatment. OWD was defined as the maintenance dose for which a patient's INR was within the target range (INR of 2-3) on $\geq 2$ consecutive laboratory measurements separated by at least 1 week. ${ }^{11}$ We used fixed dosing experience-based practices in the trial. Initial and subsequent warfarin doses were determined empirically by the physicians.

\section{Genotyping}

Genomic DNA of patients was isolated from peripheral whole blood using the QIAamp Blood Mini Kit (QIAGEN, Hilden, Germany) according to standard procedures recommended by the manufacturer, and stored at $-20^{\circ} \mathrm{C}$ until use. CYP2C9*3 (42614A $>$ C, rs1057910), VKORC1 (1639G > A, rs9923231), and CYP4F2 (18000G > A, rs2108622) polymorphisms were detected by polymerase chain reaction (PCR). Multiplex PCR conditions were optimized for SNaPshot reaction. PCR amplification was carried out in a total volume of $30 \mu \mathrm{L}$ containing $100 \mathrm{ng}$ of genomic DNA, $3 \mu \mathrm{L}$ of $10 \mathrm{X}$ PCR buffer containing $\mathrm{Mg}^{2+}, 250 \mu \mathrm{M}$ of each dNTP, $0.13 \mu \mathrm{M}$ of each primer, and $5 \mathrm{U} / \mu \mathrm{L}$ of rTaq DNA polymerase (TaKaRa, Shiga, Japan). Cycling was performed using the GeneAmp PCR system 9700 (Applied Biosystems, Foster City, CA, USA) and standard methods. ${ }^{12}$ SNaPshot Multiplex Kit (Applied Biosystems, CA, USA) was used for single nucleotide polymorphism (SNP) genotyping. Then samples were analyzed using an ABI-Prism 3130 genetic analyzer (Applied Biosystems, CA, USA). SNaPshot results were analyzed using GeneMapper ${ }^{\mathbb{B}}$ version 3.7 software (Applied Biosystems, CA, USA). Genotype classifications were as follow: VKORC1 AA and VKORC1 GA, CYP2C9*1/*1 and CYP2C9*1/*3, CYP4F2 GG and CYP4F2 GA and CYP4F2 AA. We did not find VKORC1 GG and CYP2C9*3/*3 genotypes in this cohort of Korean patients.
Statistical analysis

Categorical variables are presented as percentages, while continuous variables are presented as means \pm standard deviations. The independent t-test was used to determine the associations between GP and OWD. A generalized linear model (GLM) was used to analyze differences in OWD according to CYP2C9, VKORC1, or CYP4F2 polymorphisms. When analyzing the GLM, we adjusted the baseline characteristics and estimated the mean (standard error). Univariate analyses and multiple linear regression were performed to investigate the relationships of warfarin dose to other variables and to develop the algorithm. In this statistical modeling, the stepwise selection method was applied to identify significant clinical covari-

Table 1. Baseline characteristics of the study population

\begin{tabular}{|c|c|}
\hline Total $(n=108)$ & Numbers of patients (\%) \\
\hline Female & $56(52)$ \\
\hline \multicolumn{2}{|l|}{ Age (yr) } \\
\hline$<60$ & $46(42)$ \\
\hline $60-70$ & $58(54)$ \\
\hline$>70$ & $4(4)$ \\
\hline \multicolumn{2}{|l|}{ BMI (kg/m²) } \\
\hline$<18.5$ & $5(5)$ \\
\hline $18.5-23$ & $34(31)$ \\
\hline$>23$ & $69(64)$ \\
\hline Smoking & $11(10)$ \\
\hline Alcohol & $12(11)$ \\
\hline \multicolumn{2}{|l|}{$\operatorname{LVEF}(\%)$} \\
\hline$\geq 50$ & $78(72)$ \\
\hline$<50$ & $25(22)$ \\
\hline \multicolumn{2}{|c|}{ Main indications for treatment } \\
\hline $\mathrm{AF}$ & $83(77)$ \\
\hline PTE/DVT & $14(13)$ \\
\hline Heart valve disease & $36(33)$ \\
\hline \multicolumn{2}{|l|}{ Comorbidities } \\
\hline Cerebral infarction & $20(19)$ \\
\hline Congestive heart failure & $34(31)$ \\
\hline Hypertension & $36(33)$ \\
\hline Diabetic mellitus & $15(14)$ \\
\hline Hyperlipidemia & $6(6)$ \\
\hline \multicolumn{2}{|l|}{ Medications } \\
\hline$\beta$-blockers & $13(12)$ \\
\hline Amiodarone & $32(30)$ \\
\hline ARBs & $20(19)$ \\
\hline ACEi & $10(9)$ \\
\hline Aspirin & $16(15)$ \\
\hline Clopidogrel & $17(16)$ \\
\hline Statins & $23(21)$ \\
\hline CCBs & $20(19)$ \\
\hline Diuretics & $74(69)$ \\
\hline Nitrates & $7(6)$ \\
\hline
\end{tabular}

Values are presented as numbers (\%).

$\mathrm{BMI}$, body mass index; LVEF, left ventricular ejection fraction; $\mathrm{AF}$, atrial fibrillation; PTE, pulmonary thromboembolic disease; DVT, deep vein thrombosis; ARBs, angiotensin receptor blockers; ACEi, angiotensin-converting enzyme inhibitors; CCBs, calcium channel blockers. 
Table 2. Daily stable warfarin dose of the study patients

\begin{tabular}{|c|c|c|}
\hline Total $(n=108)$ & Warfarin dose (mg/day) & $P$-value \\
\hline \multicolumn{3}{|l|}{ Sex } \\
\hline Female & $3.1 \pm 1.5$ & 0.703 \\
\hline Male & $3.2 \pm 1.1$ & \\
\hline \multicolumn{3}{|l|}{ Age (yr) } \\
\hline$<60$ & $3.6 \pm 1.6$ & 0.001 \\
\hline $60-70$ & $2.8 \pm 0.9$ & \\
\hline$>70$ & $2.7 \pm 0.6$ & \\
\hline \multicolumn{3}{|l|}{ BMI (kg/m²) } \\
\hline$<18.5$ & $2.4 \pm 1.1$ & 0.058 \\
\hline $18.5-23$ & $2.8 \pm 1.0$ & \\
\hline$>23$ & $3.3 \pm 1.4$ & \\
\hline \multicolumn{3}{|l|}{ Smoking } \\
\hline Yes & $3.1 \pm 1.1$ & 0.971 \\
\hline No & $3.1 \pm 1.3$ & \\
\hline \multicolumn{3}{|l|}{ Alcohol } \\
\hline Yes & $3.2 \pm 1.2$ & 0.887 \\
\hline No & $3.1 \pm 1.3$ & \\
\hline \multicolumn{3}{|l|}{$\operatorname{LVEF}(\%)$} \\
\hline$\geq 50$ & $3.2 \pm 1.4$ & 0.059 \\
\hline$<50$ & $2.7 \pm 1.0$ & \\
\hline \multicolumn{3}{|c|}{ Main indications for treatment } \\
\hline \multicolumn{3}{|c|}{$\mathrm{AF}$} \\
\hline Present & $2.9 \pm 1.2$ & 0.006 \\
\hline Absent & $3.7 \pm 1.4$ & \\
\hline \multicolumn{3}{|l|}{ PTE/DVT } \\
\hline Present & $3.3 \pm 1.2$ & 0.529 \\
\hline Absent & $3.1 \pm 1.3$ & \\
\hline \multicolumn{3}{|c|}{ Heart valve disease } \\
\hline Present & $3.5 \pm 1.3$ & 0.060 \\
\hline Absent & $3.0 \pm 1.3$ & \\
\hline \multicolumn{3}{|l|}{ Comorbidities } \\
\hline \multicolumn{3}{|c|}{ Cerebral infarction } \\
\hline Present & $2.9 \pm 1.1$ & 0.314 \\
\hline Absent & $3.2 \pm 1.3$ & \\
\hline \multicolumn{3}{|c|}{ Congestive heart failure } \\
\hline Present & $2.7 \pm 1.1$ & 0.319 \\
\hline Absent & $3.3 \pm 1.3$ & \\
\hline \multicolumn{3}{|l|}{ Hypertension } \\
\hline Present & $3.1 \pm 0.9$ & 0.926 \\
\hline Absent & $3.1 \pm 1.5$ & \\
\hline \multicolumn{3}{|c|}{ Diabetic mellitus } \\
\hline Present & $3.1 \pm 1.8$ & 0.917 \\
\hline Absent & $3.1 \pm 1.2$ & \\
\hline \multicolumn{3}{|c|}{ Hyperlipidemia } \\
\hline Present & $3.5 \pm 1.2$ & 0.413 \\
\hline Absent & $3.1 \pm 1.3$ & \\
\hline \multicolumn{3}{|l|}{ Medications } \\
\hline \multicolumn{3}{|l|}{$\beta$-blockers } \\
\hline Yes & $3.0 \pm 1.1$ & 0.777 \\
\hline No & $3.1 \pm 1.3$ & \\
\hline
\end{tabular}

(Continued to the next)
Table 2. Continued

\begin{tabular}{lcc}
\hline Total $(\mathrm{n}=108)$ & Warfarin dose $(\mathrm{mg} /$ day) & $P$-value \\
\hline Amiodarone & & \\
Yes & $2.6 \pm 1.1$ & 0.012 \\
No & $3.3 \pm 1.3$ & \\
ARBs & & \\
Yes & $3.3 \pm 1.1$ & 0.548 \\
No & $3.1 \pm 1.3$ & \\
ACEi & & \\
Yes & $3.0 \pm 1.2$ & 0.769 \\
No & $3.1 \pm 1.3$ & \\
Aspirin & & \\
Yes & $3.1 \pm 1.1$ & 0.983 \\
No & $3.1 \pm 1.3$ & \\
Clopidogrel & & \\
Yes & $3.0 \pm 1.2$ & 0.729 \\
No & $3.1 \pm 1.3$ & \\
Statins & & \\
Yes & $3.0 \pm 1.2$ & 0.617 \\
No & $3.1 \pm 1.3$ & \\
CCBs & & \\
Yes & $3.0 \pm 1.2$ & 0.735 \\
No & $3.1 \pm 1.3$ & \\
Diuretics & & \\
Yes & $3.9 \pm 1.9 \pm 0.9$ \\
No & & \\
Nitrates & & \\
Yes & & \\
No & & \\
\hline
\end{tabular}

Values are presented as means \pm standard deviations.

$\mathrm{BMI}$, body mass index; LVEF, left ventricular ejection fraction; AF, atrial fibrillation; PTE, pulmonary thromboembolic disease; DVT, deep vein thrombosis; ARBs, angiotensin receptor blockers; ACEi, angiotensin-converting enzyme inhibitors; CCBs, calcium channel blockers.

ates. SPSS software 20.0 (SPSS, Inc, Chicago, IL, USA) was used for all statistical analyses. $P$-value $<0.05$ was considered to be statistically significant.

\section{Results}

Clinical characteristics

As shown in Table 1, the average age of patients was $61.5 \pm 12.4 \mathrm{yr}$, and $48 \%$ of patients were men. Only $4 \%$ of patients were older than 70 years. Most patients $(69,64 \%)$ had a high body mass index (BMI $>23$ ). Main indications for warfarin treatment were AF (77\%), PTE/ DVT (13\%), and HVD (33\%). Comorbidities were CI (19\%), CHF (31\%), hypertension (33\%), DM (14\%), and HLP (6\%). Only thirtytwo $(30 \%)$ patients reported receiving amiodarone. More patients $(74,69 \%)$ were treated with diuretics. The average OWD was $3.12 \pm$ $1.30(1-9) \mathrm{mg} /$ day. The average time percentage in the therapeutic range (TTR) in the trial was $56 \%$. Older patients ( $>70$ years) required a significantly higher OWD $(P=0.001)$. Patients diagnosed with $\mathrm{AF}$, those treated with amiodarone, and those treated with diuretics had a significantly lower OWD than other patients $(P=0.006$, $P=0.012, P=0.026$, respectively). No other clinical characteristics 
Table 3. Genotype frequencies of VKORC1, CYP2C9, and CYP4F2

\begin{tabular}{|c|c|c|c|c|c|}
\hline Gene & SNP & Allele & $\begin{array}{l}\text { Patients, } \\
\text { No. }(\%)\end{array}$ & Genotype & $\begin{array}{l}\text { Patients } \\
\text { No. (\%) }\end{array}$ \\
\hline \multirow[t]{2}{*}{ VKORC1 } & $1639 G>A$ & A & $205(94.9)$ & AA & $97(89.8)$ \\
\hline & (rs9923231) & G & $11(5.1)$ & GA & $11(10.2)$ \\
\hline \multirow[t]{2}{*}{ CYP2C9 } & $42614 \mathrm{~A}>\mathrm{C}$ & ${ }^{* 1}$ & $205(94.9)$ & ${ }^{*} 1 /{ }^{* 1}$ & $97(89.8)$ \\
\hline & (rs1057910) & *3 & $11(5.1)$ & ${ }^{*} 1 /{ }^{*} 3$ & $11(10.2)$ \\
\hline \multirow[t]{3}{*}{ CYP4F2 } & $18000 \mathrm{G}>\mathrm{A}$ & G & $147(68.1)$ & $\mathrm{GG}$ & $47(43.5)$ \\
\hline & (rs2108622) & A & 69 (31.9) & GA & $53(49.1)$ \\
\hline & & & & AA & $8(7.4)$ \\
\hline
\end{tabular}

Values are presented as numbers (\%)

SNP, single nucleotide polymorphism.

had a statistically significant effect on daily stable warfarin dose (Table 2).

Genotype frequencies of VKORC1, CYP2C9 and CYP4F2

Ninety-seven patients $(89.8 \%)$ were homozygous for the wild-type A allele of VKORC1, 11 patients (10.2\%) were heterozygous for the wild-type A allele, and no patients were homozygous for the variant $\mathrm{G}$ allele. Ninety-seven patients $(89.8 \%)$ were homozygous for CYP2C9* 1 and 11 patients $(10.2 \%)$ were heterozygous for $\mathrm{CY}$ $\mathrm{P} 2 \mathrm{C} 9 * 3$; no $* 3 / * 3$ genotypes were observed. CYP4F2 allele frequencies were $68.1 \%$ for the $\mathrm{G}$ allele and $31.9 \%$ for the A allele (Table 3).

\section{Effects of GPs on OWD}

Figure 1 summarizes the effects of GPs on OWD. VKORC1 wildtype patients (AA) had a lower OWD than variant patients (GA) ( 2.95 vs $4.63 \mathrm{mg} /$ day, respectively, $P<0.001$ ). The average OWD of patients who were CYP2C $* 1 / * 1$ was significantly higher than that of patients who were CYP2C $9 * 1 / * 3(3.30 \mathrm{vs} 1.65 \mathrm{mg} /$ day, respectively, $P<0.001)$. Mean OWD was significantly higher in patients with the CYP4F2 AA genotype than those with the GA or GG genotypes (4.40 vs. 3.12 vs $2.91 \mathrm{mg} /$ day, respectively, $P=0.014$ ).

\section{OWD model derivation}

We first used single linear recursive analysis, including OWD as the dependent variable and gender, age, BMI, habits, concomitant diseases, combined medications, LVEF, and the three genotypes as potential factors to determine the correlation between these factors and OWD. We developed an OWD prediction algorithm by including factors with a $P$ value below 0.05 in univariate analysis in multiple linear regression analysis. Multivariate analysis including seven variables with $P<0.05$ from univariate analysis (age, BMI, amiodarone, diuretics, VKORC1, CYP2C9 and CYP4F2 genotypes) was performed (Table 4, Table 5). Our model explained $41.8 \%$ of warfarin maintenance dose variability.

The equation we derived is

OWD $(\mathrm{mg} /$ day $)=4.165+1.500 \times \mathrm{VKORC} 1(\mathrm{GA})-1.115 \times \mathrm{CYP} 2 \mathrm{C} 9$ $(* 1 / * 3)-0.672 \times$ CYP4F2 (GG) $-0.791 \times$ CYP4F2 (GA) $-0.993 \times$ Age $(60-70)-0.810 \times$ Age $(>70)+0.351 *$ BMI $(18.5-23)+0.656 \times$ BMI (>23) $-0.400 \times$ Amiodarone- $0.382 \times$ Diuretics.

Coding was as follows: VKORC1 GA $=1$ if the VKORC1 genotype was GA, otherwise 0 ; CYP2C9 $* 1 / * 3=1$ if the CYP2C9 genotype was *1/*3, otherwise 0 ; CYP4F2 GG $=1$ if the CYP4F2 genotype was GG, otherwise 0; CYP4F2 GA=1 if the CYP4F2 geno-

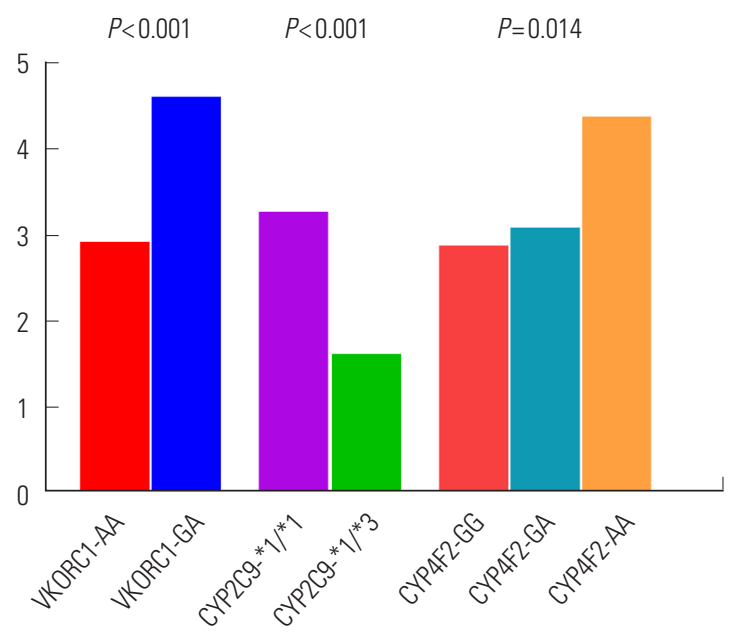

Fig. 1. Genetic polymorphisms influencing warfarin dose.

type was GA, otherwise 0 ; age in decades $=1$ for $60-70$ years, otherwise 0 ; age in decades $=1$ for $>70$ years, otherwise 0 ; BMI val$\mathrm{ue}=1$ for: $18.5-23 \mathrm{~kg} / \mathrm{m}^{2}$, otherwise $0 ;$ BMI value $=1$ for: $23 \mathrm{~kg} / \mathrm{m}^{2}$, otherwise 0 ; amiodarone status $=1$ if patient taking amiodarone, otherwise 0 ; diuretics status $=1$ if patient taking diuretics, otherwise 0 .

\section{Discussion}

The main contribution of this study was to derive an algorithm to predict OWD in Korean patients with various diseases requiring anticoagulation therapy. This algorithm was able to explain $41.8 \%$ of variation in the warfarin dose among the Korean patients enrolled in this study. We also established a definite link between multiple GPs and OWD. We demonstrated that the required dose of warfarin in Korean patients with the wild-type VKORC1 genotype (AA) was lower than that required in patients with the variant (GA) genotype (2.95 vs. $4.63 \mathrm{mg} /$ day). CYP2C9*1/*1-carriers had a significantly higher OWD than CYP2C9*1/*3-carriers (3.30 vs. $1.65 \mathrm{mg} /$ day). Although some researchers observed no significant associations between CYP4F2 polymorphisms and warfarin dose requirement in Korean and Indian patient cohorts, ${ }^{8,9}$ we found that Korean CYP4F2 AA-carriers required the highest average OWD compared to GAor GG-carriers (4.40 vs. 3.12 vs. $2.91 \mathrm{mg}$ /day, respectively, $P=0.014$ ).

It is well known that ethnicity affects OWD because of genetic variation among different ethnic populations. For example, AfricanAmericans require a higher warfarin dose than other ethnic groups. ${ }^{13}$ Warfarin maintenance dose in Asian patients is approximately 30 $40 \%$ less than that required for Caucasian patients for a similar degree of anticoagulation. These differences have partly been attributed to genetic differences in CYP2C9 and VKORC1. ${ }^{10}$ Therefore, algorithms to predict OWD that were developed in other ethnicities may not be suitable for Korean populations. A study showed that adding CYP4F2 to a model to predict warfarin dose increased the $\mathrm{R}^{2}$ value by $0.9 \%$ after adjusting for clinical and genetic variables. ${ }^{14}$ We therefore included the CYP4F2 gene in our warfarin pharmacogenetic dose prediction algorithm for Korean patients.

In a previous study that derived an algorithm to predict warfarin maintenance dose in Korean patients with AF, variables included were age, body surface area (BSA), statin status, and genetic factors (VKORC1 and CYP2C9). However, the CYP4F2 genotype was not 
Table 4. Univariate factors affecting warfarin dose

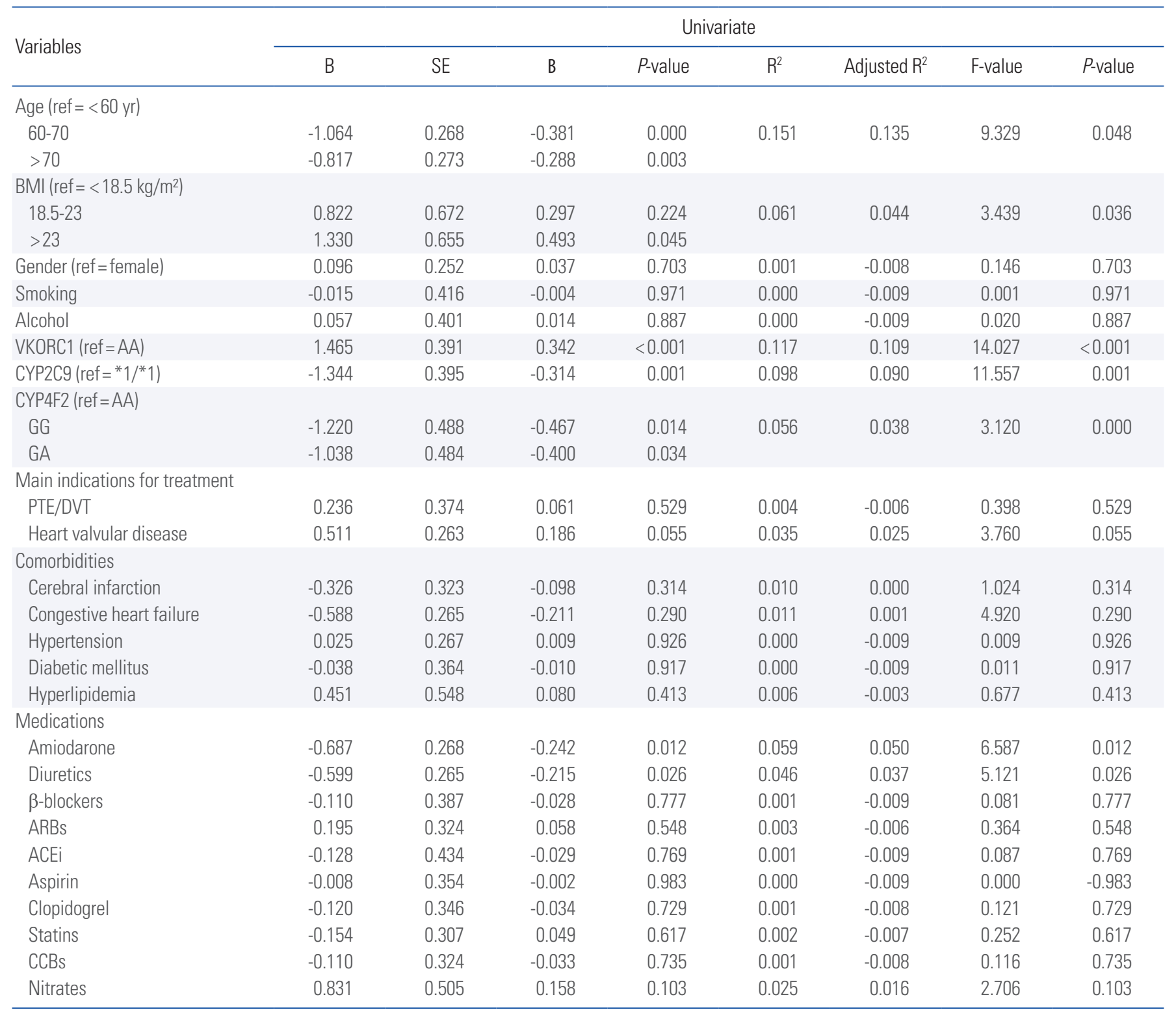

Values are presented as numbers.

BMI, body mass index; PTE, pulmonary thromboembolic disease; DVT, deep vein thrombosis; ARBs, angiotensin receptor blockers; ACEi, angiotensin-converting enzyme inhibitors; CCBs, calcium channel blockers.

included. ${ }^{15}$ In another study of Korean patients, a multiple regression model that included age, gender, BSA, INR, VKORC1, CYP2C9, CYP4F2, and GGCX polymorphisms explained $35 \%$ of the total variation in warfarin dose ${ }^{8}$ however, no co-medication information was included in the algorithm. Lee et al. suggested that CYP4F2, VKORC1, and CYP2C9 are predictive of stable warfarin doses in Korean patients with prosthetic heart valves. Their predictive algorithm included age, VKORC1, and CYP2C9 and explained 35.1\% of the variability in warfarin dose. Addition of the CYP4F2 polymorphism increased the $\mathrm{R}^{2}$ value to $38.0 \%$ for stable dose requirements. ${ }^{16}$ A multivariate analysis including non-genetic variables (age, AF) and genetic variables (genotypes of VKORC1 rs9934438, CYP2C9 rs1057910, CYP4F2 rs2108622, and UGT rs887829) explained $45.1 \%$ of the overall inter-individual variability in warfarin dose requirements in Korean patients with mechanical cardiac valves (MCV). VKORC1 genotypes accounted for $28.2 \%$ of the total variation in warfarin dose, CYP2C9 genotypes for $6.6 \%$, age for $3.0 \%$, and CYP4F2 genotypes for $1.8 \% .{ }^{17}$ In another study, an algorithm that included VKORC1, CYP2C9, CYP4F2, and vitamin D receptor (VDR) genotypes in addition to non-genetic variables explained $47.5 \%$ of the variability in stable warfarin dose in Korean patients with $\mathrm{MCV}$, and CYP4F2 explained $1.7 \%$ of inter-individual difference in overall warfarin dose. ${ }^{18}$ In our study, seven variables including age, BMI, amiodarone use, diuretic use, VKORC1, CYP2C9, and CYP4F2 polymorphisms explained $41.8 \%$ of the variance in OWD in Koreans with various diseases requiring anticoagulation therapy.

One clinical trial found that a genotype-guided dosing strategy 
Table 5. Multivariate factors affect warfarin dose

\begin{tabular}{|c|c|c|c|c|}
\hline \multirow{2}{*}{ Variables } & \multicolumn{4}{|c|}{ Multivariate } \\
\hline & B & SE & $\beta$ & $P$-value \\
\hline Interceptor & 4.165 & 0.694 & & 0.000 \\
\hline \multicolumn{5}{|l|}{ Age $(r e f=<60 \mathrm{yr})$} \\
\hline $60-70$ & -0.993 & 0.226 & -0.356 & 0.000 \\
\hline$>70$ & -0.810 & 0.228 & -0.285 & 0.001 \\
\hline \multicolumn{5}{|c|}{$\mathrm{BMI}\left(\mathrm{ref}=<18.5 \mathrm{~kg} / \mathrm{m}^{2}\right)$} \\
\hline $18.5-23$ & 0.351 & 0.549 & 0.127 & 0.524 \\
\hline$>23$ & 0.656 & 0.540 & 0.243 & 0.227 \\
\hline Diuretics & -0.382 & 0.215 & -0.135 & 0.079 \\
\hline Amiodarone & -0.400 & 0.216 & -0.143 & 0.067 \\
\hline VKORC1 (ref=AA) & 1.500 & 0.326 & 0.350 & 0.000 \\
\hline CYP2C9 $\left(\right.$ ref $\left.={ }^{*} 1 /{ }^{*} 1\right)$ & -1.115 & 0.326 & -0.260 & 0.001 \\
\hline \multicolumn{5}{|l|}{ CYP4F2 (ref=AA) } \\
\hline $\mathrm{GG}$ & -0.672 & 0.391 & -0.257 & 0.089 \\
\hline GA & -0.791 & 0.384 & -0.305 & 0.042 \\
\hline $\mathrm{R}^{2}$ & 0.472 & & & \\
\hline Adjusted $R^{2}$ & 0.418 & & & \\
\hline F-value & 8.673 & & & \\
\hline$P$-value & $<0.001$ & & & \\
\hline Durbin-Watson & 2.001 & & & \\
\hline
\end{tabular}

Values are presented as numbers.

$\mathrm{BMI}$, body mass index.

did not result in better outcomes than clinically-guided dosing. ${ }^{19}$ However, other researchers have reported that a genotype-guided algo- rithm reduced adverse events, increased anticoagulation control benefits, predicted a stable therapeutic warfarin dose, led to fewer dose adjustments, and improved accuracy and efficiency during the treatment period. ${ }^{20,21}$ We confirmed in the current study that including CYP4F2 genotype in our algorithm improved its predictive accuracy in Korean patients with a variety of diseases.

Our study had several limitations. It was conducted based on data collected exclusively from Koreans, and it was a single-center study. Our sample size was also comparatively small. Our goal in the future is to collect more patient-related information to validate the effectiveness of our algorithm and determine other factors that may affect OWD in Korean patients (such as polymorphisms in the gamma-glutamyl carboxylase gene).

In conclusion, our algorithm was able to explain $41.8 \%$ of warfarin dose differences in Korean patients with various diseases requiring anticoagulation therapy. VKORC1, CYP2C9, and CYP4F2 GPs all affected OWD in Korean patients. Although CYP4F2 polymorphisms only appear to have a mild influence on OWD, including this gene in an algorithm can improve the ability of the algorithm to accurately predict OWD.

\section{Acknowledgments}

This research was supported by Dong-A Unversity Research Fund.

\section{Conflicts of interest}

Dr. MH Kim received a research grant from Bayer Co. None of the other authors have conflicts of interest to report.

\section{References}

1. Roden DM, Johnson JA, Kimmel SE, Krauss RM, Medina MW, Shuldiner A, et al. Cardiovascular pharmacogenomics. Circ Res 2011;109:807-20.

2. Steinberg BA. How I use anticoagulation in atrial fibrillation. Blood 2016;128:2891-8.

3. Santos PCJL, Dinardo CL, Schettert IT, Soares RAG, Kawabata-Yoshihara L, Bensenor IM, et al. CYP2C9 and $\mathrm{VKORC1}$ polymorphisms influence warfarin dose variability in patients on long-term anticoagulation. Eur J Clin Pharmacol 2013;69:789-97.

4. COUMADIN ${ }^{\circledR}$ TABLETS (WARFARIN SODIUM TABLETS, USP) CRYSTALLINE https://www.accessdata.fda.gov/drugsatfda_docs/label/2016/009218s116lbl.pdf2016; Accessed February 2nd 2017.

5. Linder MW, Homme MB, Reynolds KK, Gage BF, Eby $\mathrm{C}$, Silvestrov N, et al. Interactive modeling for ongoing utility of pharmacogenetic diagnostic testing: application for warfarin therapy. Clin Chem 2009;55:1861-8.

6. Huang SW, Chen HS, Wang XQ, Huang L, Xu DL, Hu $\mathrm{XJ}$, et al. Validation of VKORC1 and CYP2C9 genotypes on interindividual warfarin maintenance dose: a prospective study in Chinese patients. Pharmacogenet Genomics 2009;19:226-34.

7. Borgiani P, Ciccacci C, Forte V, Sirianni E, Novelli L, Bramanti P, et al. CYP4F2 genetic variant (rs2108622) significantly contributes to warfarin dosing variability in the Italian population. 2009
8. Choi JR, Kim JO, Kang DR, Yoon S-A, Shin J-Y, Zhang $\mathrm{X}$, et al. Proposal of pharmacogenetics-based warfarin dosing algorithm in Korean patients. Journal of Human Genetics 2011;56:290.

9. Rathore SS, Agarwal SK, Pande S, Singh SK, Mittal T, Mittal B. CYP4F2 1347 G> A \& GGCX 12970 C > G polymorphisms: frequency in north Indians \& their effect on dosing of acenocoumarol oral anticoagulant The Indian Journal of Medical Research 2014;139:572.

10. Yin $T$ and Miyata T. Warfarin dose and the pharmacogenomics of CYP2C9 and VKORC1 - rationale and perspectives. Thrombosis Research 2007;120:1-10.

11. Lazo-Langner A, Monkman K, Kovacs M. Predicting warfarin maintenance dose in patients with venous thromboembolism based on the response to a standardized warfarin initiation nomogram. J Thromb Haemost 2009; 7:1276-83.

12. Lim YJ, Cha EY, Jung HE, Ghim JL, Lee SJ, Kim EY, et al. Genetic polymorphisms of CYP2C9, CYP2C19, CYP2D6, CYP3A4, and CYP3A5 in Vietnamese-Koreans. Translational and Clinical Pharmacology 2014; 22:70-7.

13. Cavallari LH and Perera MA. The future of warfarin pharmacogenetics in under-represented minority groups. Future Cardiology 2012;8:563-76.

14. Eriksson N, Wallentin L, Berglund L, Axelsson T, Connolly S, Eikelboom J, et al. Genetic determinants of warfarin maintenance dose and time in therapeutic treatment range: a RE-LY genomics substudy. Pharmacogenomics 2016;17:1425-39.
15. Cho HJ, On YK, Bang OY, Kim JW, Huh W, Ko JW, et al. Development and comparison of a warfarin-dosing algorithm for Korean patients with atrial fibrillation. Clin Ther 2011;33:1371-80.

16. Lee KE, Chang BC, Kim HO, Yoon IK, Lee NR, Park HY, et al. Effects of CYP4F2 gene polymorphisms on warfarin clearance and sensitivity in Korean patients with mechanical cardiac valves. Ther Drug Monit 2012; $34: 275-82$.

17. An SH, Chang BC, Lee KE, Gwak HS. Influence of UDP-Glucuronosyltransferase Polymorphisms on Stable Warfarin Doses in Patients with Mechanical Cardiac Valves. Cardiovascular Therapeutics 2015;33:324-8.

18. Lee KE, Chung JE, Yi B, Cho YJ, Kim HJ, Lee GY, et al. Influence of NR3C1 and VDR polymorphisms on stable warfarin dose in patients with mechanical cardiac valves. Int J Cardiol 2017;236:393-7.

19. Stergiopoulos K and Brown DL. Genotype-guided vs clinical dosing of warfarin and its analogues: meta-analysis of randomized clinical trials. JAMA Internal Medicine 2014; 174:1330-8

20. Baranova EV, Verhoef T, Ragia G, Le Cessie S, Asselbergs FW, de Boer A, et al. Dosing algorithms for vitamin $\mathrm{K}$ antagonists across VKORC 1 and CYP 2C9 genotypes. J Thromb Haemost 2017;15:465-72.

21. Medicine IWPCJNEJo. Estimation of the warfarin dose with clinical and pharmacogenetic data. 2009;360:75364. 